\title{
Dual Number Meadows
}

\author{
Jan A. Bergstra \\ Informatics Institute, University of Amsterdam \\ Science Park, 904, 1098 XH, Amsterdam, The Netherlands \\ j.a.bergstra@uva.nl,janaldertb@gmail.com
}

Submitted: 10 April 2019

Revised 24 May 2019

\begin{abstract}
The class of dual number meadows is introduced. By definition this class is a quasivariety. Dual number meadows contain a non-zero element the square of which is zero. These structures are non-involutive and coregular. Some properties of the equational theory of dual number meadows are discussed and an initial algebra specification is given for the minimal dual number meadow of characteristic zero which contains the dual rational numbers. Several open problems are stated.
\end{abstract}

\section{Introduction}

The idea of meadows is to introduce, on top of the signature of fields, a function symbol for inverse or for division and to insist, or prefer, that operations are total. On that basis the equational logic of known as well as novel structures and classes of structures is investigated. We call a datatype arithmetical if it models systems of numbers including well-known operators on numbers. Meadows are arithmetical datatypes which provide inverse or division or both. Within the informal notion of meadow various degrees of freedom still exist. For instance the underlying ring may or may not be commutative, inverse may or may not be an involution.

Md, the equational axiomatisation of (commutative, regular and involutive) meadows, consists of the equations for a Commutative Ring (CR) in Table 1 plus two more axioms concerning inverse: involution and regularity of Table 2. In [6] a meadow is defined as a model of Md. In section 1.2 below, however, we will provide an alternative definition of meadows which serves as a preparation for various useful generalisations of the notion of a meadow.

Regularity in combination with involution ensures that a structure which meets these requirements is the expansion of a (von Neumann) regular ring. A prominent meadow is the meadow $\mathbb{Q}_{0}$ of rational numbers. $\mathbb{Q}_{0}$ is an arithmetical datatype, and an initial algebra specification was given for it in [10], a 


$$
\begin{aligned}
(x+y)+z & =x+(y+z) \\
x+y & =y+x \\
x+0 & =x \\
x+(-x) & =0 \\
(x \cdot y) \cdot z & =x \cdot(y \cdot z) \\
x \cdot y & =y \cdot x \\
1 \cdot x & =x \\
x \cdot(y+z) & =x \cdot y+x \cdot z
\end{aligned}
$$

Table 1: CR: axioms for a commutative ring

$$
\begin{aligned}
\left(x^{-1}\right)^{-1} & =x & \text { (involution) } \\
(x \cdot x) \cdot x^{-1} & =x & \text { (regularity) } \\
\left(x^{-1} \cdot x^{-1}\right) \cdot x & =x^{-1} & (\text { coregularity) } \\
x \neq 0 \rightarrow x \cdot x^{-1} & =1 & \text { (strong regularity) }
\end{aligned}
$$

Table 2: Optional meadow properties

paper which initiated work on arithmetical datatypes with multiplicative inverse and/or division.

In this paper we will discuss some structures which come about when introducing a non-zero element $\epsilon$ for which, like for $0: x \cdot x=0$, i.e. such that $\epsilon \cdot \epsilon=0$, in the presence of a totalised inverse for multiplication, ${ }_{-}^{-1}$. Because $\epsilon$ is nonzero and has no proper inverse (i.e. no $y$ exists such that $\epsilon \cdot y=1$ ) such structures are rings but not fields.

\subsection{Preliminaries}

Some preliminaries are useful. We will briefly discuss signatures, algebras, datatypes, and abstract datatypes.

\subsubsection{Signatures}

From the theory of abstract datatypes, (see e.g. [13]), we will use the notion of a signature, that is a collection of one or more names for sorts and zero or more names for constants, with information of the sort to which these belong, plus zero or more names of functions equipped with an arity, i.e. a list of argument sorts and a target sort. In case there is just one sort, the signature is said to be single sorted otherwise it is many-sorted. Different signatures are isomorphic 
if a bijective renaming transforms the one into the other. Isomorphism classes of signatures are called similarity types, a preferred notion in logic, whereas datatype theory embodies a preference to work with signatures and to be explicit about categories of signatures when needed.

Below we will work with the following single sorted signature of rings $\Sigma_{R}$ : a single sort named $V$ for values, constant names 0 and 1 and two place functions names _ . for multiplication and _ $+_{-}$for addition and a unary function for negation. The two place function name - $_{-}$is used as an abbreviation for subtraction $x-y=x+(-y)$.

If $\Sigma$ is a single sorted signature and $\mathbb{M}$ is a structure for that signature then with $|\mathbb{M}|$ we denote the domain (alternatively: carrier) of $\mathbb{M}$. Thus if $V$ is the name of the unique sort of $\Sigma$ then $|\mathbb{M}|$ is the interpretation of $V$ that comes with $\mathbb{M}$.

\subsubsection{Algebras, datatypes, and arithmetical datatypes}

Algebras are combinations of sets and functions. An abstract datatype consists of an algebra and includes a signature which provides a name for each sort, constant, and function. Below we will assume that an algebra is a datatype and thereby is an interpretation of a signature. When considering datatypes that embody conventional number systems and variations thereof we will speak of arithmetical datatypes. An abstract datatype is the isomorphism class of a datatype, though with a fixed signature. A datatype is called minimal if it has no proper substructures.

\subsection{On the definition of meadows}

It has turned out not to be straightforward to develop a useful and flexible terminology about meadows which extends to modified but similar structures. This paper contributes to issues of nomenclature by proposing a uniform policy for defining meadow related classes of arithmetical datatypes. Instead of starting out with an axiomatic definition of meadows (models of CR enriched with an inverse function that satisfies involution and regularity) a model theoretic definition of the class of meadows is more amenable to various generalisations. We will provide the model theoretic definition of meadows and then discuss various related definitions of classes of arithmetical datatypes.

Below we will use $\hat{t}$ for the interpretation of term $t$ in a structure if it is clear from the context which structure is meant. Otherwise, when more detail is required, $t_{\mathbb{A}}$ denotes the interpretation of $t$ in $\mathbb{A}$. Moreover, we will use function symbols as well for the interpretation in a model, unless clarity requires further detail.

The signature of meadows $\Sigma_{\mathrm{Md}}$, by default with inversive notation, consists of the signature of unital rings $\left(\Sigma_{R}\right)$ extended with a unary function $-{ }^{-1}$ called inverse. A strongly regular meadow $\mathbb{A}$ is a field $\left(\left.\mathbb{A}\right|_{\Sigma_{R}}\right)$ expanded with a function $\widehat{-^{-1}}$, we interpret $-^{-1}$, in such a way that $\hat{0}^{-1}=\hat{0}$ and for all $a \neq \hat{0} \in|\mathbb{A}|$, 
$a \hat{\circ} a^{-1}=\hat{1}$. We write $E_{\text {srm }}$ for the equational theory of the class of all strongly regular meadows.

Definition 1.1. A meadow is a model of $E_{\text {srm }}$.

The idea of this definition is that it expresses that meadows are as close to fields as can be expressed with equational logic under the assumption that the inverse of 0 is 0 . It follows from results in [6], as well as from results in [17], that the model theoretic definition coincides with the axiomatic definition mentioned above. An advantage of the model theoretic definition compared with the axiomatic definition, however, is that it can be more easily generalised and adapted. Below we will list some examples of generalisations and adaptations.

For more information regarding the equational theory of meadows we refer to $[14,17,10,4]$. For recent work on the mathematical aspects of zero totalised division we mention [15].

Proposition 1.1. A strongly regular meadow is a meadow that satisfies the conditional formula: $x \neq 0 \rightarrow x \cdot x^{-1}=1$.

A strongly regular meadow is called a cancellation meadow in [4] in view of the fact that among meadows it is also characterised by the following cancellation property: $x \neq 0 \wedge x \cdot y=x \cdot z \rightarrow y=z$.

In the presence of $\mathrm{CR}$ the axiom of strong regularity (i.e. equation strong regularity in Table 2) implies regularity (equation regularity in Table 2), and under the additional assumption that $0^{-1}=0$ strong regularity also implies coregularity. Not every meadow is strongly regular. For instance the direct sum of non-trivial strongly regular meadows is not strongly regular.

Notational matters. $\Sigma(\mathbb{A})$ denotes the signature of datatype $\mathbb{A}$. For a signature $\left.\Sigma \subseteq \mathbb{A} \mathbb{A}\right|_{\Sigma}$ denotes the reduct of $\mathbb{A}$ to the signature $\Sigma$. We will use constants (e.g. 0,1 and $\epsilon$ ) also for the names of corresponding values (interpretations) in an arithmetical datatype. However, if the datatype is fixed, constants equipped with a hat (e.g. $\hat{0}$ ) always denote the interpretation of the constant, in that given datatype. When a risk of confusion is present, disambiguation by means of a subscript (e.g $1_{\mathbb{A}}$ instead of $\hat{1}$ ) indicates that the interpretation of a constant in a certain datatype or sort is meant. Below we will often use the names of functions also as names for interpretations thereof (e.g. $\hat{0}+\hat{1}$ instead of $\hat{0} \hat{+} \hat{1}$ or of $\left.0_{\mathbb{A}}+\mathbb{A} 1_{\mathbb{A}}\right)$.

\subsection{Variations on the theme of meadows}

With a variation on the theme a different but related class of structures is meant. The difference may extend to differences of the underlying signature, but also with the same signature a lot of variation is possible. In this section we discuss four known variations of the class of meadows, each of which make use of the same signature of meadows, in some cases using new constants as appealing notations for existing terms. 
Formally real meadow. A structure which satisfies all equations that are true in all strongly regular meadows which are expansions of formally real fields is a formally real meadow. We refer to [5] for further information on formally real meadows.

Skew meadow. Let a strongly regular skew meadow $\mathbb{A}$ be defined as a skew field $\left(\left.\mathbb{A}\right|_{\Sigma_{R}}\right)$ expanded with a function $\widehat{-{ }^{-1}}$, where the interpretation of $-^{-1}$, is such that $\hat{0}^{-1}=\hat{0}$ and for all $a \neq \hat{0} \in|\mathbb{A}|, a \hat{\circ} a^{-1}=\hat{1}$. Now let $E_{\text {srsm }}$ be the equational theory of the class of strongly regular skew meadows, then a skew meadow is defined as a model of $E_{\text {srsm }}$.

It is an unfortunate aspect of the terminology that a non-commutative skew meadow is not a meadow. We think that a flexible terminology about meadows is in need of such flexibility, however.

Common meadow. Common meadows, see [9] can be defined as the structures which satisfy all equations true in expansions of an extension of a field with an additional element $\mathbf{a}$ which represents an element propagating through each operation $(x+\mathbf{a}=x \cdot \mathbf{a}=-\mathbf{a}=\mathbf{a})$. Such an element is often denoted with $\perp$ in work on abstract datatypes where it is considered an error element. In the terminology of transmathematics such an element, which must be unique, is called absorptive and the preferred notation for it is $\Phi$.

Such structures with an absorptive element (say a) are expanded with a multiplicative inverse made total by setting $0^{-1}=\mathbf{a}$. Upon adopting this model theoretic definition of common meadows the results of [9] provide an alternative equational and axiomatic definition of common meadows under the additional constraint that the characteristic is zero.

Non-involutive meadow. Non-involutive meadows (see [8] ) can be defined as models of the equational theory class of expansions of fields expanded with an inverse operation which has been made total by taking $0^{-1}=f$ for some element of $f$ of the field which may not be zero. A choice with some moderate popularity is $f=1$.

\subsection{Dual numbers}

Dual numbers, as designed by William Clifford in 1873, constitute a ring extension of a field with a nilpotent element $\epsilon$ that is characterised by $\epsilon \cdot \epsilon=0$. For an introduction to dual numbers we refer to [18] and [12]. In the setting of dual numbers, like in $\mathbb{Q}_{0}$, the inverse, can be made total by requiring $1 / 0=0$.

We will turn a dual field into a so-called dual number meadow by introducing a new constant $\epsilon$ to the signature and a name for the inverse function which is made total by taking value 0 in case the inverse in the dual field is undefined. We will provide an algebraic specification of the dual number meadow $\mathbb{Q}_{0}^{d n}$. The 
result is a straightforward adaptation of the initial algebra specification of $\mathbb{Q}_{0}$ as given in [10].

\section{Strongly regular dual number meadows}

Let $\Sigma_{\mathrm{Md}, \mathrm{V}, \epsilon}$ be the signature of meadows with sort name $V$ and enriched with a constant $\epsilon$. Consider a strongly regular meadow $\mathbb{M}_{0}$. We will construct a strongly regular dual number meadow $\mathbb{M}_{0}^{d n}$ based on $\mathbb{M}_{0}$.

The carrier $V^{d n}$ of $\mathbb{M}_{0}^{d n}$ consists of the objects $(a, b)$ for $a, b \in\left|\mathbb{M}_{0}\right|$. The interpretation of constants and functions is as follows, where $0,1,{ }_{-}+_{-},{ }_{-} \cdot_{-},-_{-},{ }_{-}$, and ${ }^{-1}$ are taken from $\mathbb{M}_{0}$ :

- $\hat{0}=(0,0)$,

- $\hat{1}=(1,0)$,

- $\hat{\epsilon}=(0,1)$,

- $\hat{-}(a, b)=(-a,-b)$,

- $(a, b) \hat{+}(c, d)=(a+c, b+d)$,

- $(a, b) \hat{\cdot}(d, e)=(a \cdot d, a \cdot e+b \cdot d)$,

- $(a, b)^{\widehat{-1}}=\left(a^{-1},-b \cdot(a \cdot a)^{-1}\right)$.

Definition 2.1. For a strongly regular meadow $\mathbb{M}, \mathbb{M}_{0}^{d n}$ is a strongly regular dual number meadow, and moreover, any structure isomorphic to a structure of the form $\mathbb{M}_{0}^{d n}$ is a strongly regular dual number meadow.

As a consequence, up to isomorphism, all strongly regular dual number meadows result from this construction. Examples of strongly regular dual number meadows are $\mathbb{Q}_{0}^{d n}, \mathbb{R}_{0}^{d n}$ and $\mathbb{C}_{0}^{d n}$.

We notice that the elements of the form $(a, 0)$ for $a \in\left|\mathbb{M}_{0}\right|$ constitute a substructure which is an isomorphic copy of $\mathbb{M}_{0}$. Below we will identify $(a, 0)$ with $a$. We will also write $\mathbb{M}_{0} \oplus \epsilon \cdot \mathbb{M}_{0}$ for $\mathbb{M}_{0}^{d n}$ in order to highlight the construction of its domain.

When reasoning in an arithmetical datatype of the form $\mathbb{M}_{0} \oplus \epsilon \cdot \mathbb{M}_{0}$ for some strongly regular meadow $\mathbb{M}_{0}$, rather than working formally on the basis of axioms, we will use functions $r e(-)$ and dual $(-)$, both not mentioned in the signature, so that when $x=(a, b), r e(x)=a$ and and $\operatorname{dual}(x)=b$. These functions play a similar role to $r e(-)$ and $i m(-)$ in the case of complex numbers.

When $x=(a, b)$, upon identification of $(a, 0)$ with $a$ we may write $x=$ $r e(x)+\epsilon \cdot \operatorname{dual}(x)=a+\epsilon \cdot b$. For ease of notation, in proofs we will frequently use $a$ and $b$ as abbreviations for $r e(x)$ and $\operatorname{dual}(x)$ respectively.

Proposition 2.1. $(0, b)^{-1}=(0,0)$.

Proof. $(0, b)^{-1}=\left(0^{-1},-b \cdot(0 \cdot 0)^{-1}\right)=\left(0,-b \cdot 0^{-1}\right)=(0,-b \cdot 0)=(0,0)$. 


\section{$2.1 \quad$ Dual number meadows}

The class of dual number meadows with zero-totalised inverse is defined as follows:

Let $K^{d n}$ be the class of $\Sigma_{\mathrm{Md}, \mathrm{V}, \epsilon}$ structures of the form $\mathbb{M}_{0}^{d n}$ as defined in Paragraph 2 with $\mathbb{M}_{0}$ a strongly regular meadow. $\mathrm{Eq}_{s r d n m}$ is the equational theory of $K^{d n} \cdot \mathrm{CEq}_{s r d n m}$ is the conditional equational theory of $K^{d n}$, i.e. the set of conditional equations true in all structures in $K^{d n}$.

In Proposition 2.2 below it is shown that the conditional theory of strongly regular dual number meadows is stronger than the equational theory of that class.

Proposition 2.2. The conditional equation $\epsilon \cdot x=0 \rightarrow x^{-1}=0$ (totalisation axiom of Table 4) is valid in all strongly regular dual number meadows while it is not a consequence of $\mathrm{Eq}_{\text {srdnm }}$.

Proof. We write $\Psi_{t} \equiv \epsilon \cdot x=0 \rightarrow x^{-1}=0$ for the totalisation axiom. If totalisation is a consequence of $\mathrm{Eq}_{\text {srdnm }}$ then for some finite subset of it, say $E_{t} \subseteq E_{\text {srdnm }}, \mathrm{Eq}_{t}=\Psi_{t}$. Let $\mathbb{Q}_{0, \epsilon}$ be the expansion of $\mathbb{Q}_{0}$ with a constant $\epsilon$ which is made equal to 0 . Now consider $\mathbb{Q}_{0}^{d n}$ and consider the mapping $\phi: \mathbb{Q}_{0}^{d n} \rightarrow \mathbb{Q}_{0, \epsilon}$ defined by $\phi(a, b)=a$ (stated differently: $\phi(\epsilon)=0)$. It is easy to verify that $\phi$ is a surjective homomorphism. Because homomorphisms preserve equations, $\mathbb{Q}_{0, \epsilon} \models E_{t}$ and thus by assumption $\mathbb{Q}_{0, \epsilon}=\Psi_{t}$. Now $\mathbb{Q}_{0, \epsilon} \models \epsilon \cdot x=0 \cdot x=0$ and it follows that $\mathbb{Q}_{0, \epsilon} \models x^{-1}=0$ which is not the case. This contradiction implies that $\mathrm{Eq}_{s r d n m} \not \models \Psi_{t}$.

For that reason we define the dual number meadows as the smallest quasivariety which contains all strongly regular dual number meadows.

Definition 2.2. A dual number meadow is a structure $\mathbb{A}$ with signature $\Sigma_{\mathrm{Md}, \mathrm{V}, \epsilon}$ such that $\mathbb{A}=\mathrm{CEq}_{\text {srdnm }}$.

Definition 2.3. A weakly totalised dual number meadow is a structure $\mathbb{A}$ with signature $\Sigma_{\mathrm{Md}, \mathrm{V}, \epsilon}$ such that $\mathbb{A}=\mathrm{Eq}_{\text {srdnm }}$.

Proposition 2.2 establishes the existence of weakly totalised dual number meadows. This definition follows the pattern of definitions of meadow related classes as discussed in Paragraph 1.2 above. The following questions arise and are left open.

Problem 2.1. Does $\mathrm{Eq}_{\text {srdnm }}$ have a finite equational axiomatisation?

Problem 2.2. Does $\mathrm{CEq}_{\text {srdnm }}$ have a finite conditional equational axiomatisation?

Problem 2.3. Is $\mathrm{Eq}_{\text {srdnm }}$ decidable?

Problem 2.4. Is $\mathrm{CEq}_{\text {srdnm }}$ decidable? 


\subsection{Properties of strongly regular dual number meadows}

Leaving aside the totalised inverse, the properties of structures $\mathbb{M}_{0}^{d n}$ are wellknown, e.g. see [18]. In particular structures of the form $\mathbb{M}_{0}^{d n}$ constitute a unital commutative ring. We will refrain from checking such matters below. Moreover, these structures satisfy the following equations, each of which, except the first, are sensitive to "division by zero".

Although we are working in a model (structure algebra), for ease of notation we will write 0 instead of $\hat{0},_{-}+_{\ldots}$ instead of _ $\hat{+}_{-}$etc. We use $(a, b)$ and $(a+\epsilon \cdot b)$ as equivalent and interchangeable descriptions of the same entities.

Proposition 2.3. $\epsilon \cdot \epsilon=0$.

Proof. $(0,1) \cdot(0,1)=(0,0 \cdot 1+1 \cdot 0)=(0,0)$.

Proposition 2.4. $\epsilon^{-1}=0$.

Proof. With Proposition 2.1 immediately $(0,1)^{-1}=(0,0)$.

Proposition 2.5. $\epsilon \cdot x=0 \rightarrow x^{-1}=0$.

Proof. With $\epsilon \cdot x=(0,1) \cdot(\operatorname{re}(x), \operatorname{dual}(x))=(0, \operatorname{re}(x))$ the assumption on $x$ implies $r e(x)=0$, which implies, given that $\mathbb{M}_{0}$ is a strongly regular meadow, $x^{-1}=(\operatorname{re}(x), \operatorname{dual}(x))^{-1}=(0, \operatorname{dual}(x))^{-1}=($ with Proposition 2.1$)=0$.

Proposition 2.6. $(x \cdot y)^{-1}=x^{-1} \cdot y^{-1}$.

Proof. Let $a=\operatorname{re}(x), b=\operatorname{dual}(x), c=r e(y), d=\operatorname{dual}(y)$. Now $((a+\epsilon \cdot b) \cdot(c+$ $\epsilon \cdot d))^{-1}=(a \cdot c+\epsilon \cdot(a \cdot d+c \cdot b))^{-1}=(a \cdot c-\epsilon \cdot(a \cdot d+c \cdot b)) \cdot(a \cdot c)^{-2}=$ $((a-\epsilon \cdot b) \cdot(c-\epsilon \cdot d)) \cdot a^{-2} \cdot c^{-2}=(a+\epsilon \cdot b)^{-1} \cdot(b+\epsilon \cdot d)^{-1}$.

Proposition 2.7. If $r e(x) \neq 0$ then $x \cdot x^{-1}=1$.

Proof. With $a=\operatorname{re}(x), b=\operatorname{dual}(x):(a, b) \cdot(a, b)^{-1}=(a, b) \cdot\left(a^{-1},-b \cdot a^{-2}\right)=$ $\left(a \cdot a^{-1}, b \cdot a^{-1}-a \cdot b \cdot a^{-2}\right)=(1,0)=1$.

Proposition 2.8. $x \cdot\left(x^{-1} \cdot x^{-1}\right)=x^{-1}$.

Proof. With $a=\operatorname{re}(x), b=\operatorname{dual}(x)$ : if $r e(x)=0$ then $x^{-1}=0$ and the result is immediate. Otherwise $x \cdot\left(x^{-1} \cdot x^{-1}\right)=\left(x \cdot x^{-1}\right) \cdot x^{-1}=($ with Proposition 2.7) $=1 \cdot x^{-1}=x^{-1}$.

Proposition 2.9. $(x+\epsilon \cdot y)^{-1}=(x-\epsilon \cdot y) \cdot x^{-2}$.

Proof. Let $a=r e(x)$ and $b=\operatorname{dual}(x)$. If $a=0$ then $r e(x+\epsilon \cdot y)=0$ and with Proposition $2.1(x+\epsilon \cdot y)^{-1}=0$. Moreover $x^{-1}=0$ whence $(\mathrm{x}-\epsilon \cdot y) \cdot x^{-2}=0$. If $a \neq 0$ then we write $r e(y)=c$ and $\operatorname{dual}(y)=d$.

Now $\left.(x+\epsilon \cdot y)^{-1}=(a+\epsilon \cdot(b+y))^{-1}=(a+\epsilon \cdot b+\epsilon(c+\epsilon \cdot d))\right)^{-1}=$ $\left.(a+\epsilon \cdot b+\epsilon \cdot c+\epsilon \cdot \epsilon \cdot d))^{-1}=(a+\epsilon \cdot b+\epsilon \cdot c)\right)^{-1}=(a+\epsilon \cdot(b+c))^{-1}=$ $a^{-1}-\epsilon \cdot(b+c) \cdot a^{-2}=(a-\epsilon \cdot(b+c)) \cdot a^{-2}$. Next we consider the right hand side of the equation: $(x-\epsilon \cdot y) \cdot x^{-2}=(x-\epsilon \cdot(c+\epsilon \cdot b)) \cdot x^{-2}=$ 
$(x-\epsilon \cdot c) \cdot x^{-2}=(a+\epsilon \cdot(b-c)) \cdot(a+\epsilon \cdot b)^{-2}=(a+\epsilon \cdot(b-c)) \cdot(a-\epsilon \cdot b)^{2} \cdot a^{-4}=$ $(a+\epsilon \cdot(b-c)) \cdot\left(a^{2}-\epsilon \cdot 2 \cdot b \cdot a\right) \cdot a^{-4}=\left(a+\epsilon \cdot(b-c) \cdot(a-\epsilon \cdot 2 \cdot b) \cdot a^{-3}=\right.$ $\left(a^{2}+\epsilon \cdot a \cdot(b-c-2 \cdot b) \cdot a^{-3}=(a+\epsilon \cdot(b-c-2 \cdot b)) \cdot a^{-2}=(a-\epsilon \cdot(b+c)) \cdot a^{-2}\right.$.

Proposition 2.10. $\epsilon \cdot x=0 \rightarrow x \cdot x=0$.

Proof. Let $a=\operatorname{re}(x)$ and $b=\operatorname{dual}(x)$. Then $\epsilon \cdot x=0$ implies $a=0$. Thus $x \cdot x=(a+\epsilon \cdot b) \cdot(a+\epsilon \cdot b)=a \cdot a+\epsilon \cdot(a \cdot b+b \cdot a)=0$.

Proposition 2.11. $\epsilon \cdot x \neq 0 \rightarrow\left(x^{-1}\right)^{-1}=x$.

Proof. Let $a=\operatorname{re}(x)$ and $b=\operatorname{dual}(x)$. From $\epsilon \cdot x \neq 0$ it follows that $a \neq 0$. Now $\left(x^{-1}\right)^{-1}=\left((a+\epsilon \cdot b)^{-1}\right)^{-1}=\left(a^{-1}+\epsilon \cdot(-b) \cdot a^{-2}\right)^{-1}=$ $\left(a^{-1}\right)^{-1}+\epsilon \cdot-\left(-b \cdot a^{-2}\right) \cdot\left(a^{-1}\right)^{-2}=a+\epsilon \cdot b \cdot a \cdot a^{-1}=a+\epsilon \cdot b=x$.

Proposition 2.12. $\left(x^{-1}\right)^{-1} \cdot x^{-1}=x \cdot x^{-1}$.

Proof. Let $a=\operatorname{re}(x)$ and $b=\operatorname{dual}(x)$. If $a=0$ then $x^{-1}=0$ so both sides vanish. If $a \neq 0$ then $\epsilon \cdot x=\epsilon \cdot a \neq 0$ and Proposition 2.11 applies.

Proposition 2.13. $\left(x^{-1}\right)^{-1} \cdot x=x \cdot x$.

Proof. Let $a=r e(x)$ and $b=\operatorname{dual}(x)$. If $a=0$ then $\left(x^{-1}\right)^{-1}=0$ and $x \cdot x=0$, and the equation holds. If $a \neq 0$ then $x \cdot \epsilon \neq 0$ and Proposition 2.11 applies.

Proposition 2.14. $\epsilon \cdot x \neq 0 \rightarrow x \cdot x^{-1}=1$.

Proof. From $\epsilon \cdot x \neq 0$ it follows that $r e(x) \neq 0$. Now Proposition 2.7 applies.

Proposition 2.15. $(x+\epsilon \cdot y) \cdot(x+\epsilon \cdot y)^{-1}=x \cdot x^{-1}$.

Proof. If $\epsilon \cdot x=0$ then, by Proposition $2.5, x^{-1}=0$ and so $x \cdot x^{-1}=0$, moreover $\epsilon \cdot(x+\epsilon \cdot y)=\epsilon \cdot x=0$ so that another application of Proposition 2.5 yields the required result. If, on the other hand, $\epsilon \cdot x \neq 0$ then two applications of Proposition 2.14 suffice.

\section{Axioms for dual number meadows}

Finding axioms for a class of algebras is a somewhat arbitrary exercise. In the case of dual number meadows it is plausible to require that all axioms are satisfied in all of $K$ as defined above. This aspect amounts to the soundness of the axioms. Whether or not a finite complete set of axioms can be given is another matter (see problem 2.2 above).

In Table 3 some equational axioms for $\mathrm{Eq}_{\text {srdnm }}$ have been collected. Each of these has been proven valid in all strongly regular dual number meadows. We do not know whether or not these axioms are complete for $\mathrm{Eq}_{\text {srdnm }}$.

The conditional equation (called totalisation) of Table 4, which specifies how the inverse is made total in strongly regular dual number meadows, constitutes the only significant conditional axiom which we have found. 


$$
\begin{aligned}
\text { import } & : \mathrm{CR} \\
(x \cdot y)^{-1} & =x^{-1} \cdot y^{-1} \\
\left(x^{-1}\right)^{-1} \cdot x^{-1} & =x \cdot x^{-1} \\
\left(x^{-1}\right)^{-1} \cdot x & =x \cdot x \\
\epsilon \cdot \epsilon & =0 \\
\epsilon \cdot x \cdot x \cdot x^{-1} & =\epsilon \cdot x \\
(x+\epsilon \cdot y)^{-1} & =(x-\epsilon \cdot y) \cdot\left(x^{-1} \cdot x^{-1}\right)
\end{aligned}
$$

Table 3: $\mathbf{M d}^{d n}$ : equational axioms for a dual number meadow

$$
\begin{aligned}
\text { import } & : \mathrm{CR}, \mathrm{Md}^{d n} \\
\epsilon \cdot x=0 \rightarrow x^{-1} & =0
\end{aligned}
$$

(totalisation)

Table 4: $\mathbf{M d}^{d n, t}$

$$
\epsilon \cdot x \neq 0 \rightarrow x \cdot x^{-1}=1
$$

Table 5: Dual strong regularity 
The totalisation axiom is not true for all weakly totalising dual number meadows. Indeed the variety of weakly totalised dual number meadows contains a quasivariety (model class of the conditional equational theory $\mathrm{CEq}_{s r d n m}$ ), which better approximates the idea of dual numbers than is possible by means of equations only.

Below some consequences of the axioms will be derived. In these proofs no use is made of $r e(x)$ and dual $(x)$. The standard property of a commutative ring that $0 \cdot x=x \cdot 0=0$ will be used, however, and this fact merits a repetition of its well-known proof: $(0+0) \cdot x=0 \cdot x+0 \cdot x$, and thus $0 \cdot x+(-(0 \cdot x))=$ $(0 \cdot x+0 \cdot x)+(-(0 \cdot x))=0 \cdot x+(0 \cdot x+(-(0 \cdot x))=0 \cdot x+0=0 \cdot x$.

\subsection{Some consequences of the axioms}

The following consequences can be drawn from the axioms $\mathrm{Md}^{d n}$ of Table 3.

Proposition 3.1. $x \cdot\left(x^{-1} \cdot x^{-1}\right)=x^{-1}$.

Proof. Use axiom 14 of $\mathrm{Md}_{d}$ (taking $y=0$ ).

Proposition 3.2. $0^{-1}=0$.

Proof. In $(x+\epsilon \cdot y)^{-1}=(x-\epsilon \cdot y) \cdot\left(x^{-1} \cdot x^{-1}\right)$ (axiom 14) take $x=y=0$. Then $0^{-1}=0 \cdot 0^{-1} \cdot 0^{-1}=0$.

Proposition 3.3. $1^{-1}=1$.

Proof. In $\left(x^{-1}\right)^{-1} \cdot x=x \cdot x$ (axiom 11) take $x=1$ and obtain $\left(1^{-1}\right)^{-1}=1$. Next in $(x+\epsilon \cdot y)^{-1}=(x-\epsilon \cdot y) \cdot\left(x^{-1} \cdot x^{-1}\right)$ take $y=0$ and $x=x^{-1}$. Thus $\left(x^{-1}\right)^{-1}=x^{-1} \cdot\left(x^{-1}\right)^{-1} \cdot\left(x^{-1}\right)^{-1}$. Now, with $x=1,1=x^{-1} \cdot 1 \cdot 1$ so that $1=x^{-1}$.

Proposition 3.4. $(-1)^{-1}=-1$.

Proof. $(-1)^{-1}=$ (by Proposition 3.1$)=(-1) \cdot\left((-1)^{-1} \cdot(-1)^{-1}\right)=(-1) \cdot((-1)$. $(-1))^{-1}=(-1) \cdot(1)^{-1}=(-1) \cdot 1=-1$.

Proposition 3.5. $(-x)^{-1}=-\left(x^{-1}\right)$.

Proof. $(-x)^{-1}=((-1) \cdot x)^{-1}=(-1)^{-1} \cdot x^{-1}=(-1) \cdot x^{-1}=-\left(x^{-1}\right)$.

Proposition 3.6. $x \cdot y=1 \rightarrow y=x^{-1}$.

Proof. Assume $x \cdot y=1$ and first obtain $x^{-1} \cdot y^{-1}=1^{-1}=1$. Multiplying both sides with $x \cdot x^{-1}$ gives $x \cdot x^{-1} \cdot x^{-1} \cdot y^{-1}=x \cdot x^{-1}$, so that, with axiom 14 of $\operatorname{Md}_{d}$ (taking $y=0$ ), $x^{-1} \cdot y^{-1}=x \cdot x^{-1}$ whence $1=x \cdot x^{-1}$. Now multiplying both sides of $x \cdot y=1$ with $x^{-1}$ yields $x \cdot y \cdot x^{-1}=x^{-1}$, and with commutativity and associativity of multiplication and $1=x \cdot x^{-1}$ we obtain $y=x^{-1}$.

Proposition 3.7. $\left(\left(x^{-1}\right)^{-1}\right)^{-1}=x^{-1}$. 


$$
\begin{aligned}
1(x) & =x \cdot x^{-1} \\
x^{2} & =x \cdot x
\end{aligned}
$$

Table 6: DO: axioms for defined operators

$$
\begin{aligned}
& \text { import : } \mathrm{CR}, \mathrm{Md}^{d n}, \mathrm{DO} \\
& 1\left(x_{1}^{2}+x_{2}^{2}+x_{3}^{2}+x_{4}^{2}+1\right)=1
\end{aligned}
$$

Table 7: Initial algebra specification for rational dual numbers

Proof. $\left(\left(x^{-1}\right)^{-1}\right)^{-1}=$ (with Proposition 3.1, and associativity and commutativity of $\left.\cdot_{-}\right)=\left(\left(x^{-1}\right)^{-1}\right)^{-1} \cdot\left(\left(x^{-1}\right)^{-1}\right)^{-1} \cdot\left(x^{-1}\right)^{-1}=\left(\right.$ with axiom 9 of $\left.\mathbf{M d}^{d n}\right)$ $=\left(\left(x^{-1}\right)^{-1}\right)^{-1} \cdot\left(\left(x^{-1}\right)^{-1} \cdot x^{-1}\right)^{-1}=\left(\right.$ with axiom 10 of $\left.\mathrm{Md}^{d n}\right)=\left(\left(x^{-1}\right)^{-1}\right)^{-1}$. $\left(x \cdot x^{-1}\right)^{-1}=\left(\left(x^{-1}\right)^{-1} \cdot x\right)^{-1} \cdot\left(x^{-1}\right)^{-1}=\left(\right.$ with axiom 11 of $\left.\operatorname{Md}^{d n}\right)=(x \cdot x)^{-1}$. $\left(x^{-1}\right)^{-1}=x^{-1} \cdot x^{-1} \cdot\left(x^{-1}\right)^{-1}\left(\right.$ with axiom 10 of $\left.\mathrm{Md}^{d n}\right)=x^{-1} \cdot x^{-1} \cdot x=x^{-1}$.

In the presence of dual strong regularity:

Proposition 3.8. $\epsilon \cdot x \neq 0 \rightarrow\left(x^{-1}\right)^{-1}=x$.

Proof. With $\epsilon \cdot x \neq 0 \rightarrow x \cdot x^{-1}=1$ one finds $x \cdot x^{-1}=1$ and after application of inverse to both sides and distribution of inverse over multiplication: $x^{-1}$. $\left(x^{-1}\right)^{-1}=1$. This implies $x \cdot\left(x^{-1} \cdot\left(x^{-1}\right)^{-1}\right)=x \cdot 1$ and $\left(x \cdot x^{-1}\right) \cdot\left(x^{-1}\right)^{-1}=x$ from which one obtains $1 \cdot\left(x^{-1}\right)^{-1}=x$ and the desired identity.

Proposition 3.9. $\epsilon \cdot x \neq 0 \wedge \epsilon \cdot y \neq 0 \rightarrow \epsilon \cdot x \cdot y \neq 0$.

Proof. Suppose $\epsilon \cdot x \cdot y=0$ then $\epsilon \cdot x \cdot y \cdot x^{-1} \cdot y^{-1}=0$ and thus, with the assumptions on $x$ and $y$, and strong regularity $\epsilon \cdot x \cdot y \cdot x^{-1} \cdot y^{-1}=\epsilon \cdot x \cdot x^{-1} \cdot y \cdot y^{-1}=\epsilon \cdot 1 \cdot 1=\epsilon=0$, and thus $\epsilon \cdot x=0$ in contradiction with the assumption on $x$.

\subsection{Initial algebra specifications}

The dual fracpairs constitute a transversal for $\mathbb{Q}_{0}^{d n}$.

Definition 3.1. A dual fracpair is a closed expression of the form $p \cdot n^{-1}+\epsilon$. $q \cdot m^{-1}$ such that (i) $n, m$ are integer expressions made up from 1 and + only (i.e. $n$ and $m$ represent positive integers), (ii) $p$, and $q$ are integer expressions of the form $0, k$ or $-k$ with $k$ a positive integer expression made up from 1 and + only, and (iii) $\operatorname{gcd}(p, n)=\operatorname{gcd}(q, m)=1$.

Proposition 3.10. For positive integer expressions $n$ made up from 1 and + $\mathrm{CR}+\mathrm{Md}^{d n}+\mathrm{DO}+\mathrm{FR} 4 \vdash n \cdot n^{-1}=1$. 
Proof. Write $n$ as a sum of four squares plus 1 and apply FR4.

The following follows from the construction of $\mathbb{Q}_{0}^{d n}$.

Proposition 3.11. (i) different dual fracpairs have different interpretations in $\mathbb{Q}_{0}^{d n}$ and (ii) each element of $\mathbb{Q}_{0}^{d n}$ is the interpretation of a unique fracpair.

Theorem 3.1. The equations of Table 7 constitute a finite equational initial algebra specification of $\mathbb{Q}_{0}^{d n}$.

Proof. The soundness of the equations is a matter of inspection. To establish that the specification is an initial algebra specification of $\mathbb{Q}_{0}^{d n}$ it suffices to show that each closed expression is provably equal to a dual fracpair. The proof is done by induction on the structure of expressions. Let $t \equiv 1$ then $t=1 \cdot 1^{-1}+\epsilon \cdot 1 \cdot 1^{-1}$. If $t \equiv 0$ then $t=0 \cdot 1^{-1}+\epsilon \cdot 0 \cdot 1^{-1}$. The remaining cases are $t \equiv-r, t \equiv r^{-1}$, and $t \equiv r+s, t \equiv r \cdot s$. Let $r=p \cdot n^{-1}+\epsilon \cdot q \cdot m^{-1}$ then $t \equiv-r=-\left(p \cdot n^{-1}+\epsilon \cdot q \cdot m^{-1}\right)=(-p) \cdot n^{-1}+\epsilon \cdot(-q) \cdot m^{-1}=-[p] / n+\epsilon \cdot-[q] / m$, where $-[0] \equiv 0,-[k] \equiv-k,-[-k] \equiv k$. For the case $=r^{-1}$ three subcases on $p$ are distinguished. If $p \equiv 0$ then $t^{-1}=\left(0-\epsilon \cdot q \cdot m^{-1}\right) \cdot 0^{-2}=0=0 \cdot 1^{-1}+\epsilon \cdot 0 \cdot 1^{-1}$. If $p$ is positive, say $p=k$, then $t^{-1}=\left(k \cdot n^{-1}+\epsilon \cdot q \cdot m^{-1}\right)^{-1}=\left(k \cdot n^{-1}-\epsilon \cdot q\right.$. $\left.m^{-1}\right) \cdot\left(k \cdot n^{-1}\right)^{-2}=k \cdot n^{-1} \cdot\left(k \cdot n^{-1}\right)^{-2}+\epsilon \cdot-[q] \cdot m^{-1} \cdot\left(k \cdot n^{-1}\right)^{-2}$. Now $k \cdot n^{-1} \cdot(k \cdot$ $\left.n^{-1}\right)^{-2}=k \cdot n^{-1} \cdot k^{-1} \cdot k^{-1} \cdot\left(n^{-1}\right)^{-1} \cdot\left(n^{-1}\right)^{-1}=n^{-1} \cdot k^{-1} \cdot\left(n^{-1}\right)^{-1} \cdot\left(n^{-1}\right)^{-1}=$ $k^{-1} \cdot n^{-1} \cdot\left(n^{-1}\right)^{-1} \cdot\left(n^{-1}\right)^{-1}=k^{-1} \cdot n^{-1} \cdot n \cdot\left(n^{-1}\right)^{-1}=k^{-1} \cdot n^{-1} \cdot n \cdot n=n \cdot k^{-1}$. Next consider $-[q] \cdot m^{-1} \cdot\left(k \cdot n^{-1}\right)^{-2}$. If $q \equiv 0$ then $r=n \cdot k^{-1}+\epsilon 0 \cdot 1^{-1}$. If $q$ is positive, then $-[q] \cdot m^{-1} \cdot\left(k \cdot n^{-1}\right)^{-2}=(-q) \cdot m^{-1} \cdot k^{-1} \cdot k^{-1} \cdot\left(n^{-1}\right)^{-1} \cdot\left(n^{-1}\right)^{-1}=$ $(-q) \cdot m^{-1} \cdot k^{-1} \cdot k^{-1} \cdot\left(n^{-1}\right)^{-1} \cdot\left(n^{-1}\right)^{-1} \cdot n \cdot n^{-1}=(-q) \cdot m^{-1} \cdot k^{-1} \cdot k^{-1} \cdot\left(n^{-1}\right)^{-1}$. $n \cdot n \cdot n^{-1}=(-q) \cdot m^{-1} \cdot k^{-1} \cdot k^{-1} \cdot n \cdot n=(-a) \cdot b^{-1}$ for suitable positive integer expressions $a$ and $b$ with $\operatorname{gcd}(a, b)=1$, so that $r=n \cdot k^{-1}+\epsilon(-a) \cdot b^{-1}$. The case that $p$ is negative works similarly, and so do the notationally somewhat more involved cases for addition and multiplication.

Following [7] Theorem 2, the equation mentioned in Table 8, as a replacement of FR4 of Table 7 also provides an initial algebra specification in the case of $\mathbb{Q}_{0}$. That observation extends, eithout complications, to the context of dual rational numbers.

Proposition 3.12. The equations as mentioned Table 8 provide an equational initial algebra specification of $\mathbb{Q}_{0}^{d n}$.

In [3] it is shown that an initial algebra specification of the meadow $\mathbb{Q}_{0}$ of rationals can be given by adding to the equations $\mathrm{Md}$ of meadows a single equation with a single variable. The proof depends on some elementary consequences of Galois theory. This observation leads to the following question.

Problem 3.1. Can $\mathbb{Q}_{0}^{d n}$ be given an initial algebra specification by complementing $\mathrm{CR}+\mathrm{Md}_{d}+\mathrm{DO}$ with a single equation involving a single variable only.

Let $\mathrm{FR}=\left\{1\left(X_{1}^{2}+\cdots+X_{n}^{2}+1\right)=1 \mid n \in \mathbb{N}^{+}\right\}$. As was shown in [5] Md $+\mathrm{FR}$ constitutes a complete axiomatisation (also called a basis) of the meadow of reals $\mathbb{R}_{0}$. A corresponding question for dual number meadows reads thus: 


$$
\begin{aligned}
\text { import } & : \mathrm{CR}, \mathrm{Md}^{d n}, \mathrm{DO} \\
1\left(x_{1}^{2}+x_{2}^{2}+1\right) & =1
\end{aligned}
$$

Table 8: Formal reality for sums of two squares

Problem 3.2. Is $\mathrm{CR}+\mathrm{Md}_{d}+\mathrm{DO}+\mathrm{FR}$ a complete axiomatisation of the equational theory of $\mathbb{R}_{0}^{d n}$ ?

Problem 3.3. Is the equational theory of $\mathbb{R}_{0}^{d n}$ decidable?

\section{Discussion and concluding remarks}

In mathematics it is common practice to view numbers as elements of algebras. The algebraic perspective on such mathematical entities leads to rings, fields, the algebra of quaternions, skew fields, dual numbers, and so on. From theoretical computer science we adopt the idea that one considers datatypes rather than algebras, or algebraic structures in the presence of relations (for instance an ordering) as well as functions. A datatype consists of an algebra coupled with a signature, that is a naming scheme for sorts, constants, and functions. In this manner the signature determines a formal language and different datatypes with the same signature correspond to the various algebras that constitute different semantic models for the signature at hand. With arithmetical datatype we will refer to datatypes which contain numbers or entities similar to numbers. For an introduction to datatypes we refer to the textbook [13] and to [10] and the references mentioned in [10].

The transition from algebras to datatypes is quite significant for clarifying the relation, or rather the plurality of possible relations, between arithmetic and logic. At the same time the introduction of a signature is somehow counterintuitive from the perspective of conventional mathematics in which the notion of syntax is virtually absent. In the case of rational numbers for instance it is plausible, when contemplating an arithmetical datatype for rational numbers, to include inverse, or division, or both, in the signature. Doing so creates a setting in which $1 / 0$ is a legal expression, quite independently of its meaning. Even if division is understood as a partial function and $1 / 0$ has no defined value then still $1 / 0$ is a legal expression and the question "what is $1 / 0$ ?" is a valid question, which is entitled to an answer. The question is equally valid as the question whether or not $\Sigma_{n=1}^{\infty} 1 / n$ exists.

I consider the common practice to say that $x^{-1}$ is a notation for the unique $y$ such that $x \cdot y=1$, if it exists, is not entirely satisfactory, if only because through that convention the use of an expression already carries mathematical content as a side effect. By having inverse as a member of the signature such descriptions can be avoided. 
In the context of datatypes, equations have been identified as a very useful method of specification of classes of datatypes. Finite sets of equations can be used as proof systems amenable to automation. Given a set of equations the class of its models (algebras) has an initial algebra which is unique up to isomorphism. A finite set of equations is called an equational specification of its initial algebra. Such specifications were found for natural numbers, and integers some 50 years ago, and have since been developed for many data structures that arise in computing.

Given a finite set of equations it is often possible to find an implementation of the initial algebra by means of fully automated term rewriting techniques. In [10] a datatype for rational numbers is proposed by extending the signature with an inverse function ${ }_{-}^{-1}$ under the assumption that $0^{-1}=0$. The main result of [10] is to provide a finite equational specification of $\mathbb{Q}_{0}$, the datatype of rational numbers, with inverse made total by having $0^{-1}=0$. The question whether or not the specification of $\mathbb{Q}_{0}$ can be turned into a terminating and confluent term rewriting system has remained open, however.

The present paper provides an extension of the work in [10] to the case of dual numbers. As it turns out having $p^{-1}=0$ in case the original definitions have $p^{-1}$ undefined works quite well.

The use of equational logic opens two perspectives. On the one hand it leads to term rewriting and automatic implementation, on the other hand it leads to universal algebra where elementary classes, i.e. the model classes of sets of equations, play a key role. We define a meadow as an element of the smallest elementary class which contains all fields expanded with a zero totalised inverse operation. This approach to the definition of classes of arithmetical datatypes can be generalised in various directions, for instance dual numbers, transrational numbers, and transreal numbers.

We have thereby presented an informal format for defining classes of meadowlike structures. Technical details are provided regarding dual number meadows. In this format a definition of dual number meadow is given and some investigation of the equational theory of dual number meadows has been provided. For the case of dual rational numbers, the minimal dual number meadow of characteristic 0 , an initial algebra specification is developed. A range of open questions concerning dual number meadows has been formulated.

\subsection{Transmeadows}

In the format for defining classes of meadow-like arithmetical datatypes, as outlined in Paragraph 1.3 above, many more generalisations can be imagined.

As a new generalisation/adaptation of meadows we propose the transmeadows. These constitute a generalisation of the transrational numbers as well as of the transreal numbers ([1]) but not of the more sophisticated transcomplex numbers of [16].

The signature of transmeadows is the same as for meadows. We will first define strongly regular transmeadows. We assume that $\mathbb{A}$ is an arbitrary ordered field with domain $V$. $\mathbb{A}$ is transformed into $\mathbb{A}_{t m}$ by extending its domain and 
by defining (extending) all operations to the new domain. The domain $V$ is extended to $V_{t m}$ by introducing three pairwise distinct and new elements $\widehat{+\infty}$, $\widehat{-\infty}$, and $\widehat{\Phi}$ and expanded with a function $-\widehat{-1}$. The latter function serves as the interpretation of the interpretation of $-^{-1}$.

The elements $\widehat{+\infty}, \widehat{-\infty}$ serve as interpretations of $0^{-1},-\left(0^{-1}\right)$, and $0 \cdot 0^{-1}$ respectively.

For ease of notation constant names $+\infty,-\infty$ and $\Phi$ (the latter called nullity), are used for these entities $(\widehat{+\infty}, \widehat{-\infty}$, and $\widehat{\Phi}$ respectively). The extension of the functions of $\mathbb{A}$ to $V_{t m}$ is given by the following requirements, thereby obtaining $\mathbb{A}_{t m}$ :

- + and $\cdot$ are commutative

- $-(x \cdot y)=(-x) \cdot y,-(x+y)=(-x)+(-y)$.

- $x+\Phi=x \cdot \Phi=-\Phi=\Phi^{-1}=\Phi, 0^{-1}=+\infty, \infty^{-1}=(-\infty)^{-1}=0$, $0 \cdot \infty=\Phi,-(+\infty)=-\infty,-(-\infty)=+\infty,+\infty+(-\infty)=\Phi$,

- $x \neq 0 \wedge x \neq+\infty \wedge x \neq-\infty \wedge x \neq \Phi \rightarrow x \cdot x^{-1}=1$,

- $x \neq \Phi \wedge x \neq-\infty \rightarrow x+(+\infty)=+\infty$,

- and for all $a>0 \in V: a \cdot(+\infty)=+\infty$.

We write $\Sigma_{\mathrm{Md}_{t}}$ for the mentioned signature expansion of $\Sigma_{\mathrm{Md}}$ and we write $\mathrm{Eq}_{\text {srtm }}$ for the equational theory of the class of all strongly regular transmeadows and $\mathrm{CEq}_{\text {srtm }}$ for the conditional equational theory of the class of all strongly regular transmeadows. Here the ordering is not included in the signature and for that reason it does not occur in equations or in conditions of conditional equations.

Given these preparations a transmeadow can be defined to be a model of $\mathrm{CEq}_{\text {srtm }}$. Several questions about transmeadows remain open. We mention these questions for further research.

Problem 4.1. Is the class of transmeadows a variety (i.e. is $\mathrm{CEq}_{\text {srtm }}$ logically equivalent to $\left.\mathrm{Eq}_{\text {srtm }}\right)$ ?

Problem 4.2. Does the class of transmeadows have a finite (conditional) equational axiomatisation?

Problem 4.3. Is the equational theory of transmeadows decidable?

Problem 4.4. Find a suitable definition of dual number versions of transmeadows.

Problem 4.5. Find a suitable definition of a transcomplex meadow. 


\subsection{Concluding remarks}

We conclude with restating two older outstanding open questions concerning meadows, both of which can be reformulated for the dual number meadow of rationals as well as for the transmeadow of transrational numbers.

Problem 4.6. Is there a finite algebraic specification of $\mathbb{Q}_{0}$ which is complete for its equational theory?

Problem 4.7. Is there a finite equational initial algebra specification of $\mathbb{Q}_{0}$ which constitutes a complete (i.e. confluent and terminating) term rewrite system.

Conditional term rewriting is more involved than unconditional term rewriting and, as a consequence, a collection of conditional equations can be understood as a conditional term rewriting system in different ways. The following question represents a family of questions, corresponding to the different interpretations of systems of conditional equations as term rewrite systems.

Problem 4.8. Is there a finite conditional equational initial algebra specification of $\mathbb{Q}_{0}$ which gives rise to a complete (i.e. confluent and terminating) conditional term rewriting system.

\section{Acknowledgements}

The author acknowledges both anonymous reviewers for useful suggestions for improvements, including a simplification of the proof of Proposition 2.8, and a remark that led to the observation that (unlike the case with meadows) dual number meadows constitute a quasivariety rather than a variety.

\section{References}

[1] J.A. Anderson, N. Völker, and A. A. Adams. Perspecx Machine VIII, axioms of transreal arithmetic. Vision Geometry XV, eds. J. Latecki, D. M. Mount and A. Y. Wu, 649902; https://doi.org/10.1117/12.698153 (2007).

[2] J.A. Bergstra, I. Bethke. Note on paraconsistency and reasoning about fractions. J. of Applied Non-Classical Logics. http://dx.doi.org/ 10.1080/11663081.2015.1047232 (2015). (Also https://arxiv.org/abs/ 1410.8692.)

[3] J.A. Bergstra, I. Bethke. Subvarieties of the variety of meadows. Scientific Annals of Computer Science. vol 27 (1) 1-18, (2017). (Also https://arxiv . org/abs/1510.04021.)

[4] J. A. Bergstra, I. Bethke, and A. Ponse. Cancellation meadows: a generic basis theorem and some applications. The Computer Journal, 56 (1): 3-14, (2013). (Also https://arxiv.org/abs/0803.3969.) 
[5] J. A. Bergstra, I. Bethke, and A. Ponse. Equations for formally real meadows. Journal of Applied Logic, 13 (2): 1-23, (2015). (Also https: //arxiv.org/abs/1310.5011.)

[6] J.A. Bergstra, Y. Hirshfeld, and J.V. Tucker. Meadows and the equational specification of division. Theoretical Computer Science, 410 (12), 1261-1271 (2009).

[7] J.A. Bergstra and C.A. Middelburg. Inversive meadows and divisive meadows. Journal of Applied Logic, 9 (3): 203-220 (2011). (Also https: //arxiv.org/abs/0907.0540.)

[8] J.A. Bergstra and C.A. Middelburg. Division by zero in non-involutive meadows. Journal of Applied Logic, 13 (1): 1-12 (2015). (Also https: //arxiv.org/abs/1406.2092.)

[9] J.A. Bergstra and A. Ponse. Division by zero in common meadows. In R. de Nicola and R. Hennicker (editors), Software, Services, and Systems (Wirsing Festschrift), LNCS 8950, pages 46-61, Springer, (2015). (Also https://arxiv.org/abs/1406.6878.)

[10] J.A. Bergstra and J.V. Tucker. The rational numbers as an abstract data type. Journal of the ACM, 54 (2), Article 7 (2007).

[11] I. Bethke and P.H. Rodenburg. The initial meadows. Journal of Symbolic Logic, 75 (3), 888-895, (2010).

[12] V. Brodsky and M. Shoham. Dual numbers representation of rigid body dynamics. Mechanism and Machine Theory, 34, 693-718, (1999).

[13] H. D. Ehrich, M. Wolf, and J. Loeckx. Specification of Abstract Data Types. Vieweg + Teubner, ISBN-10:3519021153, (1997)

[14] Y. Komori. Free algebras over all fields and pseudo-fields. Report 10, pp. 9-15, Faculty of Science, Shizuoka University (1975).

[15] H. Michiwaki, S. Saitoh, and N. Yamada. Reality of the division by zero $z / 0=0$. International Journal of Applied Physics and mathematics, http://www.ijapm.org/show-63-504-1.html, DOI: 10.17706/ijapm.2015.5.3.185-191 (2016).

[16] T.S. dos Reis and J.A.D.W. Anderson. Construction of the transcomplex numbers from the complex numbers. Proc. WCECS 2014, http://www . iaeng.org/publication/WCECS2014/WCECS2014_pp97-102.pdf, (2014).

[17] H. Ono. Equational theories and universal theories of fields. Journal of the Mathematical Society of Japan, 35(2), 289-306 (1983).

[18] G.R. Veldkamp. On the use of dual numbers, vectors and matrices in instantaneous spatial kinematics. Mechanism and Machine Theory, 11, 141156 (1976). 